\title{
Factors Associate with Delirium of Liver Surgery Patients in the Intensive Care Unit of a Medical Center of Southern Taiwan
}

\author{
Huien Chun Liao', Ya Lie $\mathrm{Ku}^{2}$, Chin Tang Tu${ }^{3}$, Shu Ming Chen", Ying Chen Chiü ${ }^{5}$, Min Chung Chiang and \\ Chee Chien Yong ${ }^{7}$ \\ ${ }^{1}$ Nurse Practitioner, Liver Transplantation Team, Taiwan \\ ${ }^{2}$ Associate Professor, College of Nursing, Taiwan \\ ${ }^{3}$ Professor, Center for Teacher Education, Taiwan
}

${ }^{4}$ Assistant Professor, College of Nursing, Taiwan

${ }^{5}$ Head Nurse, Liver Transplantation Team, Taiwan

${ }^{6}$ Dean, Department of Nursing, Taiwan

${ }^{7}$ Surgery Doctor, Liver Transplantation Team, Taiwan

*Corresponding author: Ya Lie Ku, Associate Professor, College of Nursing, Taiwan

\section{ARTICLE INFO}

Received: 㓞 February 04, 2019

Published: 蔧 February 25, 2019

Citation: Huien Chun L, Ya Lie K, Chin Tang T, Shu Ming C, Ying Chen C, Min Chung C, Chee Chien Y. Factors Associate with Delirium of Liver Surgery Patients in the Intensive Care Unit of a Medical Center of Southern Taiwan. Biomed J Sci \& Tech Res 15(1)-2019. BJSTR. MS.ID.002651.

Abbreviations: ICUs: Intensive Care Units; POCD: Postoperative Cognitive Dysfunction; OLT: Orthotopic Liver Transplantation; LDLT: Living Donor Liver Transplantation; HCC: Hepatocellular Carcinoma; BUN: Blood Urea Nitrogen; Hb: Hemoglobin; RASS : Richmond Agitation and Sedation Scale; NP: Nursing Practitioner

\begin{abstract}
This is a cross-sectional and comparative study design. Before delirium-related data of the liver surgery patients were collected, a delirium assessment scale was developed by the principal investigator and one nursing practitioner (NP) in the liver ICU. The content validity of the delirium assessment scale approached 0.975 . The assessment consistencies of the Richmond Agitation and Sedation Scale, pain scores, and delirium assessment scale were evaluated by one head nurse and one NP by assessing the data of 9 liver transplant patients in the ICU; the internal consistency coefficients were $.984, .997$, and .992 , respectively. This study examined purposively sampled liver surgery patients who were hospitalized in the liver ICU of a medical center in southern Taiwan (IRB approval number 201601364B0) between November 25, 2016, and July 24, 2017. Among 34 liver surgery patients, $35.3 \%$ exhibited delirium; the average length of liver ICU stay was 12.4 days. Only the length of ICU stay was significantly correlated with delirium in the 34 liver surgery patients; therefore, a longer length of the ICU stay indicates a higher likelihood of developing delirium. In addition, the $\mathrm{Hb}$ and BUN levels were critical factors associate with delirium in the ICU liver surgery patients as well as the glucose and CRP levels were higher than the standardized levels in both delirium and non-delirium groups. Health care professionals can monitor the length of ICU stay as well as the Hb, BUN, glucose, and CRP levels to control the development of delirium in liver surgery patients in ICUs.
\end{abstract}

Keywords: Delirium; Liver Surgery; Patients; ICU

\section{Background}

Intensive care units (ICUs) have a specific and closed environment, and they provide 24-hour care to patients. Delirium is a common problem in patients of ICUs and defined as the altered state of consciousness accompanied by a change in cognition that develops over a few hours or days and tends to have a fluctuating 
course Sendelbach [1]. The incidences of delirium in the different ICU patients were reported as following. In foreign countries, according to the findings of a systematic review of 18 studies, $50 \%$ of liver transplant Italy patients whose lengths of ICU stay were $0.5-$ 32 weeks developed postoperative cognitive dysfunction (POCD) Aceto et al. [2,3]. Examined 144 adult USA patients who received liver transplantation during a period of 6 years; they revealed that delirium occurred in $25 \%$ of the patients, with the corresponding average duration being 4.56 days. Another study showed that $50 \%$ of 90 USA patients undergoing hematopoietic stem-cell transplantation developed delirium Fann et al. [4]; additionally, a study demonstrated that $10.03 \%$ of 281 Canada patients who received orthotopic liver transplantation (OLT) developed delirium in the ICU during a stay of 2 days Lescot et al. [5].

Furthermore, Wassenaar et al. [6] reported that the incidence of delirium in 2914 German patients in ICUs was $23.6 \%$. In Taiwan, Wang et al. [7,8] have reported that $58.3 \%$ and $31.5 \%$ of patients with and without drinking habits, respectively, experienced at least one episode of delirium at a hospital in central Taiwan after living donor liver transplantation (LDLT). Wang et al. [9] also reported that delirium was diagnosed in $47.4 \%$ of 37 patients after LDLT; specifically, delirium occurred approximately 7 days after surgery, and the mean duration was 5 days; the length of the ICU stay of patients with delirium was significantly longer than that of patients without delirium. Furthermore, Lin et al. [10] reported that 7.8\% of 71 patients developed delirium following hepatectomy for the treatment of hepatocellular carcinoma (HCC) at a hospital in central Taiwan. Regarding the risk factors, in foreign countries, Bhattacharya et al. [3] reported that in 144 USA patients who underwent liver transplantation, the development of delirium was associated with age, long duration of hospitalization, high likelihood of alcohol assumption, and high frequency of acquired hospital infections. Additionally, opioid doses, current and prior pain, and BUN levels were revealed to be risk factors for severe delirium in USA patients after transplantation Fann et al. [4].

Furthermore, intraoperative transfusion of packed red blood cells is also a predictor of the development of delirium in Canada patients in ICUs after OLT, which is associated with an increased length of hospital stay and mortality Lescot et al. [5]. Finally, Wassenaar et al. [6] identified nine predictors of delirium in 2914 German patients in ICUs, namely age, history of cognitive impairment, history of alcohol abuse, blood urea nitrogen (BUN), admission category, urgent admission, mean arterial blood pressure, use of corticosteroids, and respiratory status. In addition to the above risk factors, among the liver transplant patients, the incidence of neurological complications was $20 \%$, and these complications are crucial factors affecting morbidity; furthermore, hyponatremia was a risk factor for neurological complications following liver transplantation Lee et al. $[11,12]$. In addition to hyponatremia, alcohol-related Wernicke encephalopathy might be life-threatening for liver transplantat Australia patients Zuccoli et al. [13]. In Taiwan, Wang et al. [7] explored the predisposing factors for delirium in 54 ICU patients with alcoholic cirrhosis after LDLT; they reported that hepatic encephalopathy was a crucial risk factor for delirium.

On the basis of a study of 37 patients with HCC at a hospital in central Taiwan, Wang et al. [9] proposed that the risk factors associated with delirium included history of alcohol abuse and preoperative hepatic encephalopathy. Lin et al. [10] also identified that advanced age and low postoperative hemoglobin ( $\mathrm{Hb}$ ) levels were potential risk factors for postoperative delirium following hepatectomy in 71 patients with HCC at a hospital in central Taiwan. The consequences of delirium in ICU included the prolong the weaning time from ventilation and the tube insertion rate, occurrence of complications, the duration of hospitalization, care needs of the patients, medical cost, and an increase in the death Chen et al. [14-16]. Nevertheless, Olson [17] proposed that delirium is among the most common problems in ICU patients but is often overlooked or under-recognized for the critical care nurses to identify early. If the health care professionals could identify delirium in ICU patients early, they can prevent the subsequent confusion, disorientation, and unusual or irritable behaviors in the delirium patients. Consequently, the hospitalization days and death rate of ICU patients with delirium could be decreased, and the function of these patients might be improved.

\section{Purpose of the Study}

This study explored the factors associate with delirium in liver surgery patients in the ICU of a medical center in southern Taiwan.

\section{Methods}

This study used purposive samples of liver surgery patients admitted to the liver ICU of a medical center in southern Taiwan (IRB approval number 201601364B0) between November 25, 2016, and July 24, 2017. The inclusion criteria of liver surgery patients included GCS $>7$, without vision and hearing impairment, no history of dementia, drug addition, psychiatric illness, CNS diseases, and agreement of participating into the studies by the patients or family members before the surgery. The data of samples were collected when they were transferred into liver ICU after 4 hours. Although CAM-ICU has identified as the validity assessment scale for ICU delirium patients Gusmao Flores, et al. [18], the Richmond Agitation and Sedation Scale (RASS) has been reported to effectively identify different levels of delirium and correctly assess fluctuations in delirium in patients over time as well as has been also tested in 38 ICU patients Ely et al. [19]; therefore, RASS was applied to assess delirium of the liver surgery patients in this study.

Before delirium-related data were collected, a delirium assessment scale with RASS was developed by the principal investigator and one nursing practitioner (NP) in the liver ICU, and the content validity of the delirium assessment scale, which was evaluated by three ICU medical professionals and one NP, approached 0.975. The assessment consistencies of the RASS, pain scores, and delirium 
assessment scale were evaluated by one head nurse and one NP through the assessment of the data of nine liver transplant patients in the ICU. The internal consistency coefficients of the RASS, pain scores, and the delirium assessment scale were .984, .997, and .992, respectively. The delirium assessment scale was used for assessing the samples by NP twice a day during the morning at 9 am and evening at $6 \mathrm{pm}$. The rest of data in the delirium assessment scale were recorded by following the chart of each sample retrospectively.

\section{Results}

\section{Sample Characteristics}

The sample characteristics of the 34 ICU liver surgery patients with and without delirium were age, marital status, education levels, occupation, delirium, drinking habit, liver surgery, and length of ICU stay. The patients' ages were 21-75 years with average age as 51.2 years and $79.4 \%$ married. Their education ranged from the elementary level to college level, with more than $60 \%$ of the patients having graduated from high school, 32.5\% ran businesses, and $38.2 \%$ had a drinking habit. Additionally, 35.3\% of the patients exhibited delirium, and $38.3 \%$ was liver transplant patients and $61.7 \%$ were other different types of liver surgery. The length of ICU stays ranged from 1 to 27 days, with the average length being 12.4 days. Table 1 lists the demographics of the 34 liver surgery patients.

Table 1: Demographics of liver transplant patients $(\mathrm{N}=34)$.

\begin{tabular}{|c|c|c|c|}
\hline \multicolumn{4}{|c|}{ Variables } \\
\hline Age & Ranged 21-75 & Mean 51.15 & SD 13.294 \\
\hline ICU days & Ranged 1-27 & Mean 12.42 & SD 7.882 \\
\hline \multirow{5}{*}{ Marriage Status } & Married & $79.4 \%$ & \\
\hline & Non-married & $11.9 \%$ & \\
\hline & Single & $2.9 \%$ & \\
\hline & Widow & $2.9 \%$ & \\
\hline & Divorce & $2.9 \%$ & \\
\hline \multirow{3}{*}{ Education Levels } & Elementary & $11.8 \%$ & \\
\hline & High School & $64.7 \%$ & \\
\hline & College & $23.5 \%$ & \\
\hline \multirow{4}{*}{ Occupation } & Business & $32.5 \%$ & \\
\hline & Labor & $26.4 \%$ & \\
\hline & Others & $17.6 \%$ & \\
\hline & None & $23.5 \%$ & \\
\hline \multirow{2}{*}{ Drinking habit } & Yes & $38.2 \%$ & \\
\hline & No & $61.8 \%$ & \\
\hline \multirow{2}{*}{ Delirium } & Yes & $35.3 \%$ & \\
\hline & No & $64.7 \%$ & \\
\hline \multirow{2}{*}{ Liver Surgery } & Transplant & $38.3 \%$ & \\
\hline & Others & $61.7 \%$ & \\
\hline
\end{tabular}

\section{Factors Associate with Delirium}

\section{Delirium and Age, Number of Hospitalizations, Length of ICU stay}

Based on the correlation data from Table 2, delirium was not correlated with the age or number of hospitalizations of the liver surgery patients, but it was significantly correlated with the length of ICU stay. Hence, a longer length of ICU stays indicated a higher likelihood of delirium for the liver surgery patients $\left(\mathrm{r}_{\mathrm{bs}}=.39, P=.03\right)$.

Table 2: Correlation between delirium and other factors.

\begin{tabular}{|c|c|c|c|c|}
\hline & $\mathbf{1}$ & $\mathbf{2}$ & $\mathbf{3}$ & $\mathbf{4}$ \\
\hline 1.Delirium & - & & & \\
\hline 2.Age & .26 & - & & \\
\hline 3.Numbers of Hospitalization & .28 & .19 & - & \\
\hline 4. ICU days & $.39 *$ & .26 & .25 & - \\
\hline
\end{tabular}

Note: $\mathrm{P}<.05$

\section{Delirium and Drinking Habit}

Based on the data of delirium and drinking habit from (Table 3) and the chi-square test results, the 22 liver surgery patients without delirium (7 and 15 without and with drinking habits, respectively) and 12 patients with delirium ( 6 and 6 without and with drinking habits, respectively) did not show a significant relationship between delirium and drinking habit $(\chi 2=1.09, \mathrm{P}=.30)$.

Table 3: Relationship between drinking habit and delirium.

\begin{tabular}{|c|c|c|c|c|c|}
\hline & Non-Delirium & Delirium & Total & $\mathbf{\chi}^{2}$ & $\mathbf{P}$ \\
\hline Drinking habit & 15 & 6 & 21 & 1.09 & .30 \\
\hline Non-drinking habit & 7 & 6 & 13 & & \\
\hline Total & 22 & 12 & 34 & & \\
\hline
\end{tabular}

Note: $\mathrm{P}<.05$

\section{Comparison of Variables Between Delirium and Non- Delirium Groups}

When this study compared the delirium and non-delirium groups of the liver transplant recipient patients by using the independent $t$ test (Table 4), only Hb levels $(\mathrm{t}=-2.71, \mathrm{P}=.01)$ and BUN levels $(\mathrm{t}=-2.67, \mathrm{P}=.01)$ were significantly different between the two groups. The delirium group exhibited lower $\mathrm{Hb}$ and higher BUN levels than the non-delirium group. However, the count of white blood cells and levels of glucose, $\mathrm{Na}, \mathrm{K}, \mathrm{C}$-reactive protein (CRP), and creatine did not differ significantly between the groups. Nevertheless, the levels of glucose and CRP were higher than the standardized levels in both groups. 
Table 4: Comparison of variables between delirium and nondelirium groups.

\begin{tabular}{|c|c|c|c|c|c|c|}
\hline Variables & group & $N$ & $M$ & $S D$ & $t$ & $P$ \\
\hline \multirow[t]{2}{*}{ WBC } & Delirium & 12 & 8160.50 & 2198.27 & -1.48 & .15 \\
\hline & Non-Delirium & 22 & 9989.09 & 3949.09 & & \\
\hline \multirow[t]{2}{*}{$\mathrm{Hb}$} & Delirium & 12 & 8.83 & 1.37 & -2.71 & .01 \\
\hline & Non-Delirium & 22 & 10.51 & 1.89 & & \\
\hline \multirow[t]{2}{*}{ Glucose } & Delirium & 12 & 169.28 & 49.57 & 0.60 & .55 \\
\hline & Non-Delirium & 22 & 156.16 & 65.93 & & \\
\hline \multirow[t]{2}{*}{$\mathrm{Na}$} & Delirium & 12 & 139.10 & 3.02 & 1.49 & .15 \\
\hline & Non-Delirium & 22 & 137.84 & 1.91 & & \\
\hline \multirow[t]{2}{*}{$\mathrm{K}$} & Delirium & 12 & 4.11 & 1.10 & -0.45 & .66 \\
\hline & Non-Delirium & 22 & 4.46 & 2.53 & & \\
\hline \multirow[t]{2}{*}{ CRP } & Delirium & 12 & 40.55 & 20.04 & 0.31 & .76 \\
\hline & Non-Delirium & 22 & 34.91 & 61.79 & & \\
\hline \multirow[t]{2}{*}{ Bun } & Delirium & 12 & 23.91 & 16.21 & 2.67 & .01 \\
\hline & Non-Delirium & 22 & 12.76 & 8.30 & & \\
\hline \multirow[t]{2}{*}{$\mathrm{Cr}$} & Delirium & 12 & 0.68 & 0.21 & -0.47 & .64 \\
\hline & Non-Delirium & 22 & 0.75 & 0.48 & & \\
\hline
\end{tabular}

Note: $\mathrm{P}<.05$

\section{Discussion}

The incidences of delirium for the liver surgery patients in the literature from $10.03 \%$ to $58.3 \%$ based on the data of different countries depending on various types of liver surgeries and chronic illness as well as drinking behaviors and other related factors. In this study $35.3 \%$ of 34 liver surgery patients had delirium which is in the middle range of incidence according to the literature information. Additionally, the average length of ICU stay was 12.4 days of 34 liver surgery patients in this study which is longer than the hospitalization days comparing with the literature data ranged from 2-7days. The reason for the liver surgery patients in this study stayed in the liver ICU longer than the rest of cases in the literature is because there are many liver transplant patients in this study comparing with the other studies in the literature. Liver transplant is a big and complex surgery process for patients to justify their bodies to fit into the new organs into their own systems that should take more time to adapt.

Because the patients in this study were aged $<65$ years, age was not significantly correlated with delirium. However, most studies have identified age as a risk factor for delirium. Half of the patients were businessmen with drinking habits due to their work environment; nevertheless, drinking habit was not significantly correlated with delirium in this study because of the small sample sizes and lower percentage of 34 liver patients who had drinking habits. In addition, $38.2 \%$ of liver surgery patients in this study with drinking habits were not necessary to be the alcoholism; even studies have indicated that alcohol consumption is a significant factor associate with delirium in liver surgery patients. Furthermore, the results regarding Na levels are also different from those in previous studies that have indicated that hyponatremia was a risk factor for neurological complications following liver transplantation Lee et al. [11,12]. In this study, only the levels of $\mathrm{Hb}$ and BUN were critical factors correlated with delirium; these findings are similar to those of previous studies Fann et al. [4-6,10].

The universal and consistent finding is that the longer lengths of ICU stay were significantly correlated with the development of delirium in liver surgery patients. This finding is similar to those of Wang et al. [9], who revealed that 37 HCC patients with delirium had a significantly longer length of ICU stay than patients without delirium. In addition, Bhattacharya et al. [3] reported that the development of delirium in 144 USA patients who received liver transplantation was associated with long lengths of hospitalization and a high frequency of acquired hospital infections. The liver transplant patients with longer lengths of ICU stay were considerably more likely to have had hospitalized infection, which also caused an increase in their blood sugar levels. In this study, the glucose and CRP levels were higher than the standardized levels for patients who stayed in the liver ICU. However, the higher glucose and CRP for the liver surgery patients could be the medication of steroid after surgery to maintain the functions of major organs in the whole system of the liver surgery patients.

\section{Conclusion and Suggestions}

In conclusion, according to the findings of this study, among 34 liver surgery patients, $35.3 \%$ had delirium, and the average length of ICU stay was 12.4 days. Only the length of ICU stay was significantly correlated with delirium in the 34 liver surgery patients, which indicates that liver surgery patients with long lengths of ICU stay might easily develop delirium. Additionally, the levels of $\mathrm{Hb}$ and BUN are critical factors associate with delirium for the liv- 
er surgery patients in ICUs. However, in this study, the glucose and CRP levels of the liver surgery patients were higher than the standardized levels in the delirium and non-delirium groups. Health care professionals could monitor the length of ICU stay and levels of $\mathrm{Hb}$, BUN, glucose, and CRP for controlling delirium of liver surgery patients in ICUs. In addition to data monitoring, psychiatric and psychosocial assessments and interventions after liver surgery are crucial for the quality of care outcomes Grover et al. [20] because $50 \%$ of liver transplant patients developed POCD, according to the findings of a systematic review of 18 studies Aceto et al. [2]. The authors suggest that health care professionals should develop non-pharmacological nursing interventions for solving the psychiatric and psychosocial problems of liver surgery patients instead of using pharmacological procedures.

\section{References}

1. Sendelbach S, Guthrie PF (2009) Acute confusion/delirium: Identification, assessment, treatment, and prevention. Journal of Gerontology Nursing 35(11): 11-18.

2. Aceto P, Perilli V, Lai C, Ciocchetti P, Vitale F, et al. (2015) Postoperative cognitive dysfunction after liver transplantation. General Hospital Psychiatry 37(2): 109-115.

3. Bhattacharya B, Maung A, Barre K, Maerz L, Rodriguez Davalos MI, et al. (2017) Postoperative delirium is associated with increased intensive care unit and hospital length of stays after liver transplantation. Journal of Surgical Research 207: 223-228.

4. Fann JR, Hubbard RA, Alfano CM, Roth Roemer S, Katon WJ, et al. (2011) Pre-and post-transplantation risk factors for delirium onset and severity in patients undergoing hematopoietic stem-cell transplantation. Journal of Clinical Oncology 29(7): 895-901.

5. Lescot T, Karvellas CJ, Chaudhury P, Tchervenkov J, Paraskevas S, et al. (2013) Postoperative delirium in the intensive care unit predicts worse outcomes in liver transplant recipients. Cancer Journal of Gastroenterology 27(4): 207-212.

6. Wassenaar A, van den Boogaard M, van Achterberg T, Slooter AJC, Kuipe MA, et al. (2015) Multinational development and validation of an early prediction model for delirium in ICU patients. Intensive Care Medicine 4(6): 1048-1056.

7. Wang SH, Hsieh CE, Wang JY, Lin HC, Lin ZZ, et al. (2012) Predisposing factors of delirium for alcoholic liver cirrhosis with living donot liver transplantation in the intensive care units. Taiwan Critical Care Medicine 13: $178-184$

8. Wang SH, Hsieh CE, Wang JY, Lin HC, Lin PY, et al. (2013) Factors associated with delirium in intensive care units of non-alcoholic cirrhotic patients who received living donor liver transplantation. Formosan Journal of Medicine 17(6): 610-616.

9. Wang SH, Wang JY, Lin PY, Lin KH, Ko CJ, et al. (2014) Predisposing risk factors for delirium in living donor liver transplantation patients in intensive care units. PLOS ONE 9(5): e96676.

10. Lin LZ, Hsieh CE, Lin HC, Wang SH, Lin KH, et al. (2014) Risk factors for postoperative delirium following hepatectomy in patients with hepatocellular carcinoma. Formosan Journal of Medicine 18(4): 390396.

11. Lee J, Kim DK, Lee JW, Oh KH, Oh YK, et al. (2013) Rapid correction rate of hyponatremia as an independent risk factor for neurological complication following liver transplantation. The Tohoku Journal of Experimental Medicine 229(2): 97-105.

12.Živković AA, Abdel Hamid H (2010) Neurologic manifestations of transplant complications . Neurologic Clinics 28(1): 235-251.

13. Zuccoli G, Siddiqui N, Gravo I, Bailey A, Gallucci M, Massimo Gallucci, et al. (2010) Neuroimaging findings in alcohol-related encephalopathies. American Journal of Roentgenology 195(6): 1378-1384.

14. Chen YL, Sun SG, Huang SW (2012) Delirium among surgical intensive care unit patients. Changhua Nursing 19(4): 33-40.

15. Fu FH, Shi SF, Chang YC (2007) Acute confusion among intensive care unit patients. Yuan Nursing 1(2): 52-63.

16. Wu SL, Gao FZ, Ming Y (2007) Assessment and nursing care of patients in acute confusion in intensive care units. Journal of Nursing 54(6): 67-72.

17. Olson T (2012) Delirium in the intensive care unit: role of the critical care nurse in early detection and treatment. Dynamics 23(4): 32-36.

18. Gusmao Flores D, Salluh JIF, Chalhub RA, Quarantini LC (2012) The confusion assessment method for the intensive care unit (CAM-ICU) and intensive care delirium screening checklist (ICDSC) for the diagnosis of delirium: a systematic review and meta-analysis of clinical studies. Critical Care 16(R115): 1-10.

19. Ely EW, Truman B, Shintani A, Thomason JW, Wheeler AP, et al. (2003) Monitoring sedation status over time in ICU patients: reliability and validity of the Richmond Agitation-Sedation Scale (RASS). JAMA 289(22): 2983-2991.

20. Grover S, Sarkar S (2012) Liver transplant-psychiatric and psychosocial aspects. Journal of Clinical and Experimental Hepatology 2(4): 382-392.

\section{ISSN: 2574-1241}

\section{DOI: 10.26717/BJSTR.2019.15.002651}

Ya Lie Ku. Biomed J Sci \& Tech Res

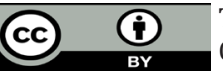

This work is licensed under Creative

Commons Attribution 4.0 License

Submission Link: https://biomedres.us/submit-manuscript.php

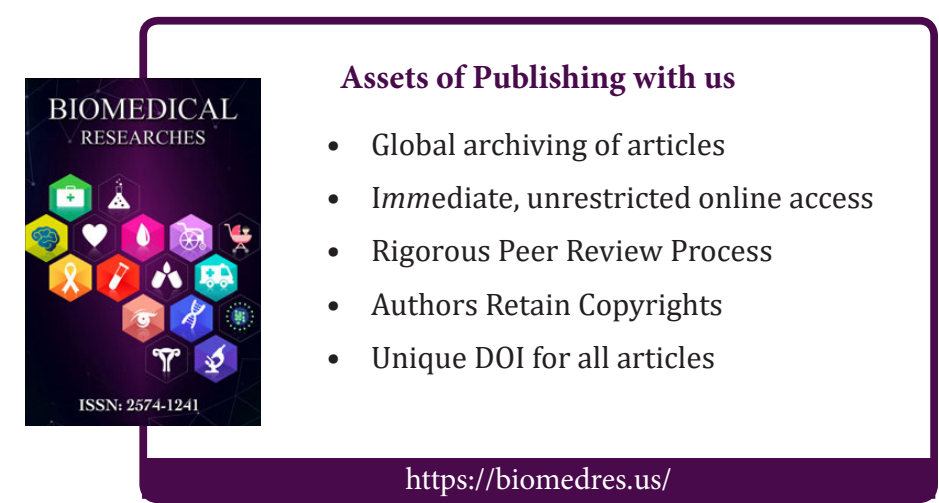

